

\title{
A formalism for equivariant Schubert calculus
}

\begin{abstract}
Dan Laksov
In previous work we have developed a general formalism for Schubert calculus. Here we show how this theory can be adapted to give a formalism for equivariant Schubert calculus consisting of a basis theorem, a Pieri formula and a Giambelli formula. Our theory specializes to a formalism for equivariant cohomology of grassmannians. We interpret the results in a ring that can be considered as the formal generalized analog of localized equivariant cohomology of infinite grassmannians.
\end{abstract}

\section{Introduction}

Schubert calculus, in the form of the cohomology, or Chow ring, of a Grassmann variety $\operatorname{Grass}^{l}(n)$ of $n-l$-planes in $n$-space, has a long and important history. Recently much of the story has been extended to the equivariant cohomology ring, which has a much richer structure, being an algebra over a polynomial ring with $n$ generators. One knows generators and relations for this algebra, and formulas for Schubert classes, which form a basis.

In this article we give a different and more general perspective on these algebras. The idea comes from a previous article [Laksov and Thorup 2009] where a generalized Schubert calculus is considered as the ring of symmetric polynomials $A\left[T_{1}, \ldots, T_{l}\right]^{\mathrm{sym}}$ in $l$ variables over an arbitrary ring $A$ acting on the exterior product $\bigwedge_{A}^{l} A[T]$ of the polynomial ring $A[T]$ in one variable (see also [Gatto 2005] and [Gatto and Santiago 2009]).

In the present article we show how this formalism, when expressed in terms of the basis of $A[T]$ consisting of generalized factorial powers

$$
(T \mid y)^{i}=\left(T-y_{1}\right) \cdots\left(T-y_{i}\right)
$$

for given elements $y_{1}, y_{2}, \ldots$ in A, gives a general equivariant Schubert calculus consisting of a basis theorem, a Pieri formula and a Giambelli formula. The theory

MSC2000: primary 14N15; secondary 57R91, 14M15.

Keywords: equivariqant cohomology, Schubert calculus, quantum cohomology, symmetric polynomials, exterior products, Pieri's formula, Giambelli's formula, GKM condition, factorial Schur functions, grassmannians. 
is further specialized for each $n$ in [Laksov 2008] (which builds upon the results of the present article) to give the equivariant Schubert calculus for $\operatorname{Grass}^{l}(n)$, or more generally for the $l$-quotients $\operatorname{Grass}_{S}^{l}(\mathscr{E})$ of a locally free $\mathscr{O}_{S}$-module on any scheme $S$ with a bivariant intersection theory. In Section 6 of [Laksov 2008] we gave a detailed account of the geometric interpretation of $A\left[T_{1}, \ldots, T_{l}\right]^{\mathrm{sym}}$, and in Section 7 we showed how the theory of the present article can be used to recover the quantum and equivariant quantum cohomology of grassmannians.

The geometry whose cohomology we are generalizing can be realized as the union of the grassmannians $\operatorname{Grass}^{l}(n)$, as $n$ goes to infinity, taken over the natural embedding of $\operatorname{Grass}^{l}(n)$ in $\operatorname{Grass}^{l}(m)$, for $n<m$, equivariant with respect to a natural embedding of $\mathrm{GL}(n)$ in $\mathrm{GL}(m)$. The surjections to equivariant cohomology of $\operatorname{Grass}^{l}(n)$ for each $n$ can be constructed using the description of equivariant cohomology that comes from localization, so that an element of the equivariant cohomology is given by specifying a polynomial at each fixed point of the torus $\left(\mathbb{C}^{*}\right)^{n}$, subject to the conditions made explicit by M. Goresky, R. Kottwitz and R. MacPherson, and called GKM conditions.

In our generalization the connection between the above construction and the description of equivariant cohomology coming from localization is given, in the case when $A$ is a polynomial ring $\mathbb{Z}\left[y_{1}, y_{2}, \ldots\right]$ in independent variables $y_{1}, y_{2}, \ldots$, via an $A\left[T_{1}, \ldots, T_{l}\right]^{\mathrm{sym}}$-module isomorphism

$$
\bigwedge_{A}^{l} A[T] \rightarrow H(l),
$$

where $H(l)$ is the $A$-algebra consisting of elements that are of bounded total degree in the variables $y_{1}, y_{2}, \ldots$ and satisfy the GKM condition in the product $\prod_{\lambda \in\left\{\begin{array}{c}\infty \\ l\end{array}\right\}} A$ of $A$ taken over all lists $\lambda: \lambda_{1} \lambda_{2} \ldots$ of ones and zeros with exactly $l$ zeros, with coordinatewise addition and multiplication. The ring $H(l)$ can be thought of as the graded limit of the equivariant cohomologies of the grassmannians $\operatorname{Grass}^{l}(n)$, as $n$ goes to infinity.

The observation that equivariant cohomology could be interpreted within the framework of exterior powers was made in [Gatto and Santiago 2006] and [Santiago 2006]. In the latter reference it was proved that there exists an isomorphism between Schubert calculus on exterior powers, that is, Schubert calculus in a setting similar to the $\operatorname{Grass}^{l}(n)$ case mentioned above, and equivariant cohomology for Grassmann manifolds, and for simple examples (projective space $k=1$, and the Knutson-Tao [2003] example with $k=2, n=4$ ) it was indicated what the isomorphism should look like; see [Gatto and Santiago 2006]. It was this work that inspired us to consider the equivariant cohomology of Grassmann schemes and to describe the explicit isomorphism in the general case.

We note that we obtain a generalization of the full equivariant Pieri formula, and not only the Chevalley formula for divisors (see, for example, [Knutson and Tao 
2003; Mihalcea 2006; Lakshmibai et al. 2006; Kostant and Kumar 1986; Molev and Sagan 1999; Okun'kov and Ol'shanskii 1997] for various forms of the latter formula). This general form was first given by T. Santiago and we essentially reproduce the calculations of [Santiago 2006] in our language. A full version is also obtained by S. Robinson [2002] by different methods.

Our adaption of the general Schubert calculus to the equivariant case is built upon factorial Schur functions used by L. C. Mihalcea [2006; 2008] to describe the equivariant quantum cohomology ring of grassmannians (see [Arabia 1989; Billey 1999; Lascoux 2003], for earlier related work on double Schubert polynomials and complete flag varieties). We develop the theory of factorial Schur functions in such a way that specialization of our formalism directly gives the Goresky-KottwitzMacPherson description of equivariant cohomology coming from localization (see also [Knutson and Tao 2003; Arabia 1989; Kostant and Kumar 1986]), and the theory of L. C. Mihalcea [2006; 2008]. It should be pointed out that our equivariant Giambelli formula is a generalization of an unshifted version of that of [Mihalcea 2008], that is, it uses only generalized factorial powers and not their shifted counterparts. A different, but similar, equivariant Giambelli formula is given in [Lakshmibai et al. 2006]. All versions specialize to the classical Giambelli formula [Fulton 1998; 1997]. Fulton [2007, Lecture 7] explains how the equivariant Giambelli formula for grassmannians amounts to a degeneracy formula [Kempf and Laksov 1974] in algebraic geometry.

\section{Exterior powers and residues}

In this section we interpret the main results of [Laksov and Thorup 2009] in terms of factorial Schur functions. Our general version of equivariant Schubert calculus is the general Schubert calculus interpreted using polynomials of the form

$$
\left(T-y_{1}\right) \cdots\left(T-y_{i}\right)
$$

for elements $y_{1}, y_{2}, \ldots$ in $A$. These polynomials form the building blocks of the approach to equivariant quantum Schubert calculus by Mihalcea. To facilitate the understanding of the correspondence between our theory and Mihalcea's we use the notation of [Macdonald 1995, I §3 Example 20], which is also used in [Mihalcea 2008].

Notation 1.1. All rings in the following will be commutative with a unit. Let $A$ be such a ring. All exterior powers and tensor products will be taken with respect to $A$. We denote by $A[T]$ and $A\left[T_{1}, \ldots, T_{l}\right]$ the polynomial rings over $A$ in 1 , respectively $l$, independent variables. The symmetric functions in $A\left[T_{1}, \ldots, T_{l}\right]$ we denote by $A\left[T_{1}, \ldots, T_{l}\right]^{\text {sym }}$. We identify the tensor product $\bigotimes_{A}^{l} A[T]$ with $A\left[T_{1}, \ldots, T_{l}\right]$ and consider $\bigotimes_{A}^{l} A[T]$ as a module over $A\left[T_{1}, \ldots, T_{l}\right]^{\text {sym }}$ via this 
identification. The starting point of our interpretation of Schubert calculus is the easily verified observation that the $A\left[T_{1}, \ldots, T_{l}\right]^{\mathrm{sym}}$-module structure on the tensor product $\bigotimes_{A}^{l} A[T]$ induces an $A\left[T_{1}, \ldots, T_{l}\right]^{\text {sym }}$-module structure on the exterior power $\bigwedge_{A}^{l} A[T]$ via the canonical surjection $\bigotimes_{A}^{l} A[T] \rightarrow \bigwedge_{A}^{l} A[T]$; [Laksov and Thorup 2009, Section 1].

Let $e_{i}=\cdots+b_{i,-l} T^{-l}+b_{i,-l+1} T^{-l+1}+\cdots+b_{i,-1} T^{-1}+\cdots$ for $i=1, \ldots, l$ be a collection of Laurent series with coefficients $b_{i, j}$ in a ring. We write, as in [Laksov and Thorup 2009, 0.3],

$$
\operatorname{Res}\left(e_{1}, \ldots, e_{l}\right):=\operatorname{det}\left(b_{i,-j}\right)=\left(\begin{array}{cccc}
b_{1,-1} & b_{1,-2} & \cdots & b_{1,-l} \\
b_{2,-1} & b_{2,-2} & \cdots & b_{2,-l} \\
\vdots & \vdots & \ddots & \vdots \\
b_{l,-1} & b_{l,-2} & \cdots & b_{l,-l}
\end{array}\right) .
$$

Let $y_{1}, y_{2}, \ldots$ be elements in $A$ and write

$$
(T \mid y)^{i}=\left(T-y_{1}\right) \cdots\left(T-y_{i}\right) \text { for } i=0,1, \ldots
$$

The polynomials $(T \mid y)^{0},(T \mid y)^{1}, \ldots$ are called generalized factorial powers and form a basis for the $A$-module $A[T]$. Let $Q(T)=\left(T-T_{1}\right) \cdots\left(T-T_{l}\right)$, and let $b: b_{1} \geq \cdots \geq b_{l} \geq 0$ be a partition. We write

$$
s_{b}\left(T_{1}, \ldots, T_{l} \mid y\right)=\operatorname{Res}\left((T \mid y)^{b_{1}+l-1} / Q(T), \ldots,(T \mid y)^{b_{l}} / Q(T)\right) .
$$

The polynomials $s_{b}\left(T_{1}, \ldots, T_{l} \mid y\right)$ we refer to as factorial Schur functions; [Macdonald 1995, I §3 Example 20].

We now rewrite the Main result 0.5 of [Laksov and Thorup 2009] in the basis $(T \mid y)^{0},(T \mid y)^{1}, \ldots$ of $A[T]$.

Theorem 1.2. Let $Q(T)=\left(T-T_{1}\right) \cdots\left(T-T_{l}\right)$. For every collection $f_{1}, \ldots, f_{l}$ of polynomials in $A[T]$ and for every partition $b: b_{1} \geq \cdots \geq b_{l} \geq 0$ we have:

(1) (Poincaré duality) The $A\left[T_{1}, \ldots, T_{l}\right]^{\mathrm{sym}}$-module $\bigwedge_{A}^{l} A[T]$ is free of rank one with generator $(T \mid y)^{l-1} \wedge \cdots \wedge(T \mid y)^{0}$.

(2) (The determinantal formula)

$$
f_{1} \wedge \cdots \wedge f_{l}=\operatorname{Res}\left(f_{1} / Q, \ldots, f_{l} / Q\right)\left((T \mid y)^{l-1} \wedge \cdots \wedge(T \mid y)^{0}\right) .
$$

(3) (The equivariant Giambelli-Gatto formula)

$$
(T \mid y)^{b_{1}+l-1} \wedge \cdots \wedge(T \mid y)^{b_{l}}=s_{b}\left(T_{1}, \ldots, T_{l} \mid y\right)\left((T \mid y)^{l-1} \wedge \cdots \wedge(T \mid y)^{0}\right) .
$$


Proof. Since we clearly have $(T \mid y)^{l-1} \wedge \cdots \wedge(T \mid y)^{0}=T^{l-1} \wedge \cdots \wedge T^{0}$ the first and second assertion are equivalent to the first and second assertions of [Laksov and Thorup 2009, 0.5].

Assertion (3) is a particular case of assertion (2).

Notation 1.3. For every collection $f_{1}, \ldots, f_{l}$ of elements in $A[T]$ we write

$$
\left(f_{i}\left(T_{j}\right)\right)=\left(\begin{array}{ccc}
f_{1}\left(T_{1}\right) & \cdots & f_{1}\left(T_{l}\right) \\
\vdots & & \vdots \\
f_{l}\left(T_{1}\right) & \cdots & f_{l}\left(T_{l}\right)
\end{array}\right),
$$

and we let $\Delta=\prod_{1 \leq i<j \leq l}\left(T_{i}-T_{j}\right)=\operatorname{det}\left(T_{j}^{l-i}\right)$. The polynomial $\operatorname{det}\left(f_{i}\left(T_{j}\right)\right)$ is divisible by $\Delta$ because it is alternating in $T_{1}, \ldots, T_{l}$.

Proposition 1.4. Let $Q(T)=\left(T-T_{1}\right) \cdots\left(T-T_{l}\right)$. For every collection of polynomials $f_{1}, \ldots, f_{l}$ in $A[T]$ we have

$$
\operatorname{Res}\left(f_{1} / Q, \ldots, f_{l} / Q\right)=\operatorname{det}\left(f_{i}\left(T_{j}\right)\right) / \Delta .
$$

In particular, for every partition $b: b_{1} \geq \cdots \geq b_{l} \geq 0$ we have

$$
\begin{aligned}
s_{b}\left(T_{1}, \ldots, T_{l} \mid y\right)=\operatorname{Res}\left((T \mid y)^{b_{1}+l-1} / Q\right. & \left.\ldots,(T \mid y)^{b_{l}} / Q\right) \\
& =\operatorname{det}\left(\left(T_{j} \mid y\right)^{b_{i}+l-i}\right) / \operatorname{det}\left(\left(T_{j} \mid y\right)^{l-i}\right) .
\end{aligned}
$$

Proof. Both sides of the first equality of the proposition are multilinear and alternating in $f_{1}, \ldots, f_{l}$. Hence it suffices to prove the equality when $f_{i}=T^{h_{i}+l-i}$ with $h_{1} \geq \cdots \geq h_{l}$. An easy calculation (see [Laksov and Thorup 2009, 0.6]) shows that we then have an equality $\operatorname{Res}\left(f_{1} / Q, \ldots, f_{l} / Q\right)=s_{h_{1}, \ldots, h_{l}}\left(T_{1}, \ldots, T_{l}\right)$, where the polynomials $s_{h_{1}, \ldots, h_{l}}\left(T_{1}, \ldots, T_{l}\right)=\left(s_{h_{i}-i+j}\right)$, with $s_{i}$ the $i$-th complete symmetric function in $T_{1}, \ldots, T_{l}$, are the ordinary Schur functions (see [Macdonald 1995, I $\S 3]$, for example). However, by the Jacobi-Trudi formula (see [Macdonald 1995, I $\S 3$ (3.4)], for example) we have $s_{h_{1}, \ldots, h_{l}}\left(T_{1}, \ldots, T_{l}\right)=\operatorname{det}\left(T_{j}^{h_{i}+l-i}\right) / \operatorname{det}\left(T_{j}^{l-i}\right)$.

The following result indicates a different approach to the determinantal formula from that presented in [Laksov and Thorup 2009].

Proposition 1.5. There is an isomorphism of $A\left[T_{1}, \ldots, T_{l}\right]^{\mathrm{sym}}$-modules

$$
\sigma^{\mathrm{sym}}: \bigwedge_{A}^{l} A[T] \rightarrow A\left[T_{1}, \ldots, T_{l}\right]^{\mathrm{sym}}
$$

determined by mapping $f_{1} \wedge \cdots \wedge f_{l}$ to $\operatorname{det}\left(f_{i}\left(T_{j}\right)\right) / \Delta$.

Proof. The existence of the homomorphism follows since $\operatorname{det}\left(f_{i}\left(T_{j}\right)\right) / \Delta$ is multilinear and alternating in $f_{1}, \ldots, f_{l}$.

To prove that the homomorphism is $A\left[T_{1}, \ldots, T_{l}\right]^{\mathrm{sym}}$-linear it suffices to prove that the homomorphism $\sigma: \otimes_{A}^{l} A[T] \rightarrow A\left[T_{1}, \ldots, T_{l}\right]^{\text {sym }}$ determined by $\sigma\left(f_{1} \otimes\right.$ 
$\left.\cdots \otimes f_{l}\right)=\operatorname{det}\left(f_{i}\left(T_{j}\right)\right) / \Delta$ is $A\left[T_{1}, \ldots, T_{l}\right]^{\text {sym }}$-linear. We first note that we have an equality $\sigma\left(\left(T_{1}^{h_{1}} \cdots T_{l}^{h_{l}}\right) f_{1}\left(T_{1}\right) \cdots f_{l}\left(T_{l}\right)\right)=\operatorname{det}\left(T_{j}^{h_{i}} f_{i}\left(T_{j}\right)\right) / \Delta$. Fix a permutation $\tau$ of $[1, l]$. The contribution to $\operatorname{det}\left(T_{j}^{h_{i}} f_{i}\left(T_{j}\right)\right)$ from the elements in rows $1, \ldots, l$ and the corresponding columns $\tau(1), \ldots, \tau(l)$ is $T_{\tau(1)}^{h_{1}} \cdots T_{\tau(l)}^{h_{l}} f_{1}\left(T_{\tau(1)}\right) \cdots f_{l}\left(T_{\tau(l)}\right)$. Let $f$ be the sum of the elements $T_{\tau(1)}^{k_{1}} \cdots T_{\tau(l)}^{k_{l}}$ taken over all the different $l$ tuples $\left(k_{1}, \ldots, k_{l}\right)$ that are permutations of $\left(h_{1}, \ldots, h_{l}\right)$. Then the contribution to $\sigma\left(f\left(f_{1}\left(T_{1}\right) \otimes \cdots \otimes f_{l}\left(T_{l}\right)\right)\right)$ from the elements in rows $1, \ldots, l$ and corresponding columns $\tau(1), \ldots, \tau(l)$ is equal to $f f_{1}\left(T_{\tau(1)}\right) \cdots f_{l}\left(T_{\tau(l)}\right)$. Consequently we have $\sigma\left(f\left(f_{1} \otimes \cdots \otimes f_{l}\right)\right)=f \operatorname{det}\left(f_{i}\left(T_{j}\right)\right) / \Delta$. The homomorphism $\sigma^{\text {sym }}$ is $A\left[T_{1}, \ldots, T_{l}\right]^{\mathrm{sym}}$-linear since all symmetric functions are linear combinations of polynomials of the form $f$.

It follows from Theorem 1.2(1) that $\bigwedge_{A}^{l} A[T]$ is a free $A\left[T_{1}, \ldots, T_{l}\right]^{\mathrm{sym}}$-module of rank one with generator $(T \mid y)^{l-1} \wedge \cdots \wedge(T \mid y)^{0}$. Since this generator maps to 1 by $\sigma^{\text {sym }}$ and $\sigma^{\text {sym }}$ is an $A\left[T_{1}, \ldots, T_{l}\right]^{\text {sym }}$-module homomorphism it follows that $\sigma^{\text {sym }}$ is an isomorphism.

\section{Strings, partitions and factorial Schur functions}

In this section we give the main properties of factorial Schur functions. This will provide us with the natural foundation for the treatment of the generalization of the description of equivariant cohomology given by Goresky-Kottwitz-MacPherson (see also [Knutson and Tao 2003; Arabia 1989; Kostant and Kumar 1986]) and of the theory of Mihalcea, both mentioned above. To facilitate the understanding of the correspondence between the theories we have conformed to the notation of [Knutson and Tao 2003] as much as possible.

Notation 2.1. Denote by $\left\{\begin{array}{c}\infty \\ l\end{array}\right\}$ all strings $\lambda: \lambda_{1} \lambda_{2} \ldots$ consisting of zeros and ones, with exactly $l$ zeros. We consider $\left\{\begin{array}{c}\infty \\ l\end{array}\right\}$ as a lattice with inequality $\lambda^{\prime} \geq \lambda$ if $\sum_{i=1}^{j} \lambda_{i}^{\prime} \geq \sum_{i=1}^{j} \lambda_{i}$ for $j=1,2, \ldots$

An inversion in $\lambda$ is a pair $(i, j)$ with $i<j$ such that $1=\lambda_{i}>\lambda_{j}=0$. Denote by $\operatorname{inv}(\lambda)$ the inversions in $\lambda$ and write $l(\lambda)=|\operatorname{inv}(\lambda)|$.

We introduce a similar terminology and notation for partitions. Let $\left\{\begin{array}{c}\infty \\ l\end{array}\right\}_{\mathscr{P}}$ consist of all partitions $b: b_{1} \geq \cdots \geq b_{l} \geq 0$. We consider $\left\{\begin{array}{c}\infty \\ l\end{array}\right\}_{\mathscr{P}}$ as a lattice with inequality $b^{\prime} \geq b$ when $b_{i}^{\prime} \geq b_{i}$ for $i=1, \ldots, l$. To each partition $b: b_{l} \geq \cdots \geq b_{l} \geq 0$ we associate a strictly decreasing sequence $a_{1}>\cdots>a_{l}>0$, where $a_{j}=b_{j}+l-j+1$ for $j=1, \ldots, l$.

An inversion in $b$ is a pair $\left(i, a_{j}\right)$ such that $i<a_{j}$ and $i \notin\left\{a_{j+1}, \ldots, a_{l}\right\}$. We denote the inversions in $b$ by $\operatorname{inv}(b)$ and write $l(b)=|\operatorname{inv}(b)|$. Clearly $l(b)=$ $\sum_{i=1}^{l} b_{i}$.

Let $0<a(\lambda)_{l}<\cdots<a(\lambda)_{1}$ be the positions where the zeros appear in $\lambda$ : $\lambda_{1} \lambda_{2} \ldots$ for $\lambda$ in $\left\{\begin{array}{c}\infty \\ l\end{array}\right\}$, that is $\lambda_{a(\lambda)_{i}}=0$ for $i=1, \ldots, l$. We obtain a partition 
$b(\lambda): b(\lambda)_{1} \geq \cdots \geq b(\lambda)_{l} \geq 0$ in $\left\{\begin{array}{c}\infty \\ l\end{array}\right\}_{\mathscr{P}}$, with $a(\lambda)_{j}=b(\lambda)_{j}+l-j+1$ for $j=1, \ldots, l$, and

$b(\lambda)_{i}=\{$ the number of ones to the left of zero number $l-i+1$ in the string $\lambda\}$.

Example. Take $l=5$. Consider the sequence $\lambda=01001010111 \ldots$ Then the sequence $a(\lambda)$ is equal to 13468 , and the sequence $b(\lambda)$ to 32110 . More geometrically, one can record the sequence $\lambda$ by a planar path, read from southwest to northeast, starting from $(0,0)$, and ending on the line $y=l$, by making zeros correspond to vertical steps and ones to horizontal steps. Then $b(\lambda)$ is the partition whose boundary is traced by $\lambda$.

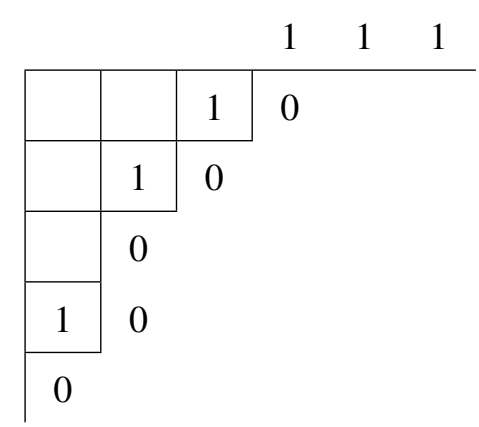

It is clear that $\operatorname{inv}(\lambda)=\operatorname{inv} b(\lambda)$ and thus $l(\lambda)=\sum_{i=1}^{l} b(\lambda)_{i}=l(b(\lambda))$.

From a partition $b: b_{1} \geq \cdots \geq b_{l} \geq 0$ we obtain, conversely, a string $\lambda(b)$ : $\lambda(b)_{1} \lambda(b)_{2} \ldots$ in $\left\{\begin{array}{c}\infty \\ l\end{array}\right\}$ defined by $\lambda(b)_{a_{i}}=0$ for $i=1, \ldots, l$, the remaining $\lambda(b)_{i}$ being one.

Lemma 2.2. There is a length preserving bijection of lattices between partitions $\left\{\begin{array}{c}\infty \\ l\end{array}\right\}_{\mathscr{P}}$ and $\left\{\begin{array}{c}\infty \\ l\end{array}\right\}$ that maps $b$ to $\lambda(b)$, and $\lambda$ to $b(\lambda)$.

Proof. It is obvious from the definitions that the map described in the lemma gives a bijection between $\left\{\begin{array}{c}\infty \\ l\end{array}\right\}_{\mathscr{P}}$ and $\left\{\begin{array}{c}\infty \\ l\end{array}\right\}$, and we observed above that the map preserves length.

That the map is a homomorphism of lattices follows since $b(\lambda)_{i}$ and $b\left(\lambda^{\prime}\right)_{i}$ are the number of ones to the left of zero number $l-i+1$ in $\lambda$, respectively $\lambda^{\prime}$.

Lemma 2.3. Let $\lambda, \lambda^{\prime}$ be strings in $\left\{\begin{array}{c}\infty \\ l\end{array}\right\}$, and let $b, b^{\prime}$ be the corresponding partitions in $\left\{\begin{array}{c}\infty \\ l\end{array}\right\}_{\mathscr{P}}$.

(1) When $\lambda$ and $\lambda^{\prime}$ differ only in the $i$-th and $j$-th positions and $(i, j) \in \operatorname{inv}(\lambda)$, then $j=a(\lambda)_{p}$ and $i=a\left(\lambda^{\prime}\right)_{q}$ for some $p$ and $q$, and the remaining elements in the sequences $a(\lambda)_{1}>\cdots>a(\lambda)_{l}$ and $a\left(\lambda^{\prime}\right)_{1}>\cdots>a\left(\lambda^{\prime}\right)_{l}$ are the same.

(2) When $b$ and $b^{\prime}$ are such that the sequences $a_{l}<\cdots<a_{1}$ and $a_{l}^{\prime}<\cdots<a_{1}^{\prime}$ differ only where $j=a_{p}$ and $i=a_{q}$ with $i<j$, then the strings $\lambda(b)$ and $\lambda\left(b^{\prime}\right)$ differ only in the positions $i$ and $j$, and $(i, j) \in \operatorname{inv}(\lambda(b))$. 
Proof. Assertion (1) follows since the zeros in $\lambda$ are in the same positions as the zeros in $\lambda^{\prime}$ except in positions $i$ and $j$ where $\lambda_{j}=0$ and $\lambda_{i}^{\prime}=0$.

Similarly assertion (2) follows since the zeros in $\lambda(b)$ and $\lambda\left(b^{\prime}\right)$ are in the same positions except zero number $j$ in $\lambda(b)$ and zero number $i$ in $\lambda\left(b^{\prime}\right)$.

In the next two results we give the main properties of factorial Schur functions.

Theorem 2.4 (Vanishing Theorem; see also [Molev and Sagan 1999]). For every partition $b: b_{1} \geq \cdots \geq b_{l} \geq 0$ we have:

(1) $s_{b}\left(y_{b_{1}+l}, \ldots, y_{b_{l}+1} \mid y\right)=\prod_{(i, j) \in \operatorname{inv}(b)}\left(y_{j}-y_{i}\right)$.

(2) Let $h: h_{1} \geq \cdots \geq h_{l} \geq 0$ be a partition that is not greater than or equal to $b$. Then $s_{b}\left(y_{h_{1}+l}, \ldots, y_{h_{l}+1} \mid y\right)=0$.

Proof. We may assume that $A$ is the polynomial ring $\mathbb{Z}\left[y_{1}, y_{2}, \ldots\right]$ in the variables $y_{1}, y_{2} \ldots$ because, once the theorem is proved in this case, we can, for general $A$, specialize the variables $y_{1}, y_{2}, \ldots$ to any sequence of elements in $A$.

(1) The $(p, q)$-th entry in $\left(\left(T_{j} \mid y\right)^{b_{i}+l-i}\right)$ is $\left(T_{q} \mid y\right)^{b_{p}+l-p}=\prod_{i=1}^{b_{p}+l-p}\left(T_{q}-y_{i}\right)$. If $p<q$ we have $b_{q}+l-q+1 \leq b_{p}+l-p$, and thus $\left(T \mid y_{b_{q}+l-q+1}\right)^{b_{p}+l-p}=0$.

When $p=q$ we have $\left(T \mid y_{b_{p}+l-p+1}\right)^{b_{p}+l-p}=\prod_{i=1}^{b_{p}+l-p}\left(y_{b_{p}+l-p+1}-y_{i}\right)$. Consequently the matrix $\left(T \mid y_{b_{j}+l-j+1}\right)^{b_{i}+l-i}$ is lower triangular, and it follows from what we just saw and from Notation 2.1 that the product of the diagonal elements divided by $\prod_{1 \leq i<j \leq l}\left(y_{b_{i}+l-i+1}-y_{b_{j}+l-j+1}\right)$ is $\prod_{(i, j) \in \operatorname{inv}(\lambda(b))}\left(y_{j}-y_{i}\right)$.

(2) By assumption $h_{p}<b_{p}$ for some $p$ and thus $h_{p}+l-p+1<b_{p}+l-p+1$. Then, for $i \leq p$ and $p \leq j$ we have $h_{j}+l-j+1 \leq h_{p}+l-p+1<b_{p}+$ $l-p+1 \leq b_{i}+l-i+1$. Consequently $\left(T \mid y_{h_{j}+l-j+1}\right)^{b_{i}+l-i}=0$. Thus the $(p \times(l-p+1))$-matrix in the upper right corner of $\left(\left(T \mid y_{h_{j}+l-j+1}\right)^{b_{i}+l-i}\right)$ is zero, and thus $\operatorname{det}\left(\left(T \mid y_{h_{j}+l-j+1}\right)^{b_{i}+l-i}\right)=0$. Since $\prod_{1 \leq i<j \leq l}\left(y_{b_{i}+l-i+1}-y_{b_{j}+l-j+1}\right)$ is not a zero divisor in $\mathbb{Z}\left[y_{1}, y_{2}, \ldots\right]$ we have $s_{b}\left(y_{h_{1}+l}, \ldots, y_{h_{l}+1} \mid y\right)=0$.

The next result will be used in Section 3 to describe equivariant Schubert calculus as presented above. It will imply the main properties of Schubert classes generalizing the description Goresky-Kottwitz-MacPherson in the notation of [Knutson and Tao 2003]. We stress that the methods used to prove parts (2) and (3) of the next result are similar to those used by Knutson and Tao to prove corresponding results for Schubert classes (proof of Lemma 1 in Section 2.1 and of Proposition 1 in Section 2.4). We could have chosen the opposite approach and used the results of Knutson and Tao to obtain information on factorial Schur functions. It is however, more in the spirit of this work to focus on the properties of factorial Schur functions.

Theorem 2.5. Let $A=\mathbb{Z}\left[y_{1}, y_{2}, \ldots\right]$ be the polynomial ring in the independent variables $y_{1}, y_{2}, \ldots$, and let $g \in A\left[T_{1}, \ldots, T_{l}\right]^{\mathrm{sym}}$. Moreover, let $\left\{\begin{array}{c}\infty \\ l\end{array}\right\}_{k}$ be the subset of $\left\{\begin{array}{c}\infty \\ l\end{array}\right\}_{\mathscr{P}}$ consisting of partitions $h: h_{1} \geq \cdots \geq h_{l} \geq 0$ with $h_{1} \leq k$. 
(1) $g$ satisfies the GKM (Goresky-Kottwitz-MacPherson) condition. That is:

When $b$ and $b^{\prime}$ are partitions in $\left\{\begin{array}{c}\infty \\ l\end{array}\right\}_{\mathscr{P}}$ such that the sequences $b_{l}+1<$ $\cdots<b_{1}+l$ and $b_{l}^{\prime}+1<\cdots<b_{1}^{\prime}+l$ differ only where the first sequence is equal to $j$ and the second is equal to $i$ with $i \neq j$, then $g\left(y_{b_{l}+1}, \ldots, y_{b_{1}+l}\right)-$ $g\left(y_{b_{l}^{\prime}+1}, \ldots, y_{b_{1}^{\prime}+l}\right)$ is divisible by $y_{i}-y_{j}$.

(2) Let $g=\sum_{h \in \mathscr{I}} z_{h} s_{h}\left(T_{1}, \ldots, T_{l} \mid y\right)$ with $z_{h} \in A$ and with $\Phi \subseteq\left\{\begin{array}{c}\infty \\ l\end{array}\right\}_{k}$, and assume that $g\left(y_{h_{1}+l}, \ldots, y_{h_{l}+1}\right)=0$ for all partitions $h$ in $\left\{\begin{array}{c}\infty \\ l\end{array}\right\}_{k}$. Then $z_{h}=0$ for all $h \in \mathscr{I}$.

(3) For a given partition b, conditions (1) and (2) of Theorem 2.4 characterize the homogeneous symmetric functions. More precisely and more generally:

We have equality $g\left(y_{c_{1}+l}, \ldots, y_{c_{l}+1}\right)=s_{b}\left(y_{c_{1}+l}, \ldots, y_{c_{l}+1} \mid y\right)$ for all $c \in$ $\left\{\begin{array}{c}\infty \\ l\end{array}\right\}_{k}$ when the following three conditions are fulfilled:

(a) For every partition $b \in\left\{\begin{array}{c}\infty \\ l\end{array}\right\}_{k}$ we have an equality $g\left(y_{b_{1}+l}, \ldots, y_{b_{l}+1}\right)=$ $\prod_{1 \leq i<j \leq \operatorname{inv}(b)}\left(y_{j}-y_{i}\right)$.

(b) $g\left(y_{h_{1}+l}, \ldots, y_{h_{l}+1}\right)=0$ for all partitions $h \in\left\{\begin{array}{c}\infty \\ l\end{array}\right\}_{k}$ that are not greater than or equal to $b$

(c) For all $c$ in $\left\{\begin{array}{c}\infty \\ l\end{array}\right\}_{k}$ we have that $g\left(y_{c_{1}+l}, \ldots, y_{c_{l}+1}\right)$ is homogeneous in $y_{1}, y_{2}, \ldots$ of degree $l(b)$.

Proof. Assertion (1) is clear since $g$ is symmetric in $T_{1}, \ldots, T_{l}$.

To prove assertion (2) we assume that some $z_{h}$ is non-zero and choose $b$ minimal such that $z_{b} \neq 0$. If $z_{h} \neq 0$ we must then have $b_{p}<h_{p}$ for some $p$. It follows from Theorem 2.4(2) that $s_{h}\left(y_{b_{1}+l}, \ldots, y_{b_{l}+1} \mid y\right)=0$. Consequently we have that $g\left(y_{b_{1}+l}, \ldots, y_{b_{l}+1}\right)$ is equal to the sum $\sum_{h \in \Phi} z_{h} s_{h}\left(y_{b_{1}+l}, \ldots, y_{b_{l}+1} \mid y\right)=$ $z_{b} s_{b}\left(y_{b_{1}+l}, \ldots, y_{b_{l}+1} \mid y\right)$ which is non-zero by Theorem 2.4(1). This contradicts the assumption of (2) and thus proves that $z_{h}=0$ for all $h \in \mathscr{I}$.

We now prove assertion (3). Let $g$ satisfy the conditions (i)-(iii) for some $b$. Assume that $g\left(y_{h_{1}+l}, \ldots, y_{h_{l}+1}\right)$ is not equal to $s_{b}\left(y_{h_{1}+l}, \ldots, y_{h_{l}+1} \mid y\right)$ for some $h \in\left\{\begin{array}{c}\infty \\ l\end{array}\right\}_{k}$ and let $c$ be a minimal element in $\left\{\begin{array}{c}\infty \\ l\end{array}\right\}_{k}$ with the property that

$$
\left(g-s_{b}\left(T_{1}, \ldots, T_{l} \mid y\right)\right)\left(y_{c_{1}+l}, \ldots, y_{c_{l}+1}\right) \neq 0
$$

Then $c \geq b$; otherwise it follows from assumption (ii) and from Theorem 2.4(2) that $g\left(y_{c_{1}+l}, \ldots, y_{c_{l}+1}\right)=0=s_{b}\left(y_{c_{1}+l}, \ldots, y_{c_{l}+1} \mid y\right)$. Moreover $c$ is strictly bigger than $b$, since $\left(g-s_{b}\left(T_{1}, \ldots, T_{l} \mid y\right)\right)\left(y_{b_{1}+l}, \ldots, y_{b_{l}+1}\right)=0$ by assumption (i) and by Theorem 2.4(1). In particular, $l(c)>l(b)$. But from the GKM condition 2.5(1) it follows that $\prod_{(i, j) \in \operatorname{inv}(c)}\left(y_{j}-y_{i}\right)$ divides $\left(g-s_{b}\left(T_{1}, \ldots, T_{l} \mid y\right)\right)\left(y_{c_{1}+l}, \ldots, y_{c_{l}+1}\right)$. This is impossible since $\left(g-s_{b}\left(T_{1}, \ldots, T_{l} \mid y\right)\right)\left(y_{c_{1}+l}, \ldots, y_{c_{l}+1}\right)$ is homogeneous of degree $l(b)$ in $y_{1}, y_{2}, \ldots$ by assumption (iii) of (3). We have thus a contradiction showing that there is an equality $g\left(y_{c_{1}+l}, \ldots, y_{c_{l}+1}\right)=s_{b}\left(y_{c_{1}+l}, \ldots, y_{c_{l}+1} \mid y\right)$ for all $c \in\left\{\begin{array}{c}\infty \\ l\end{array}\right\}_{k}$, and we have proved assertion (3). 


\section{Factorial Schur functions and Schubert classes}

In this section we present the generalization of the Goresky-Kottwitz-MacPherson description of equivariant cohomology alluded to several times above. We also give the precise correspondence between this formalism and the general Schubert calculus interpreted via factorial Schur functions.

Notation 3.1. Let $A=\mathbb{Z}\left[y_{1}, y_{2}, \ldots\right]$ be the polynomial ring in the variables $y_{1}, y_{2}$, $\ldots$ over $\mathbb{Z}$. We denote by $\prod_{\lambda \in\left\{\begin{array}{l}\infty \\ l\end{array}\right\}} A$ all lists $\alpha=(\alpha \mid \lambda)$ of elements in $A$. This is a ring with componentwise multiplication, and the unit is the list with $1 \mid \lambda=1_{A}$ for all $\lambda$. We consider $\prod_{\lambda \in\left\{\begin{array}{c}\infty \\ l\end{array}\right\}} A$ as an $A$-algebra mapping $a \in A$ to the list $\alpha_{a}$ with $\alpha_{a} \mid \lambda=a$ for all $\lambda$, and we define an $A$-algebra homomorphism

$$
\sigma^{\text {equ }}: A\left[T_{1}, \ldots, T_{l}\right]^{\mathrm{sym}} \rightarrow \prod_{\lambda \in\left\{\begin{array}{c}
\infty \\
l
\end{array}\right\}} A
$$

by $\sigma^{\text {equ }}\left(f\left(T_{1}, \ldots, T_{l}\right)\right)=\alpha_{f}$ where $\alpha_{f} \mid \lambda=f\left(y_{b(\lambda)_{1}+l}, \ldots, y_{b(\lambda)_{l}+1}\right)$.

For each partition $b: b_{1} \geq \cdots \geq b_{l} \geq 0$ in $\left\{\begin{array}{c}\infty \\ l\end{array}\right\}_{\mathscr{\rho}}$ and for each string $\lambda: \lambda_{1} \lambda_{2} \ldots$ in $\left\{\begin{array}{c}\infty \\ l\end{array}\right\}$ we write

$$
S_{b}=\sigma^{e q u}\left(s_{b}\left(T_{1}, \ldots, T_{l} \mid y\right)\right) \quad \text { and } \quad S_{\lambda}=S_{b(\lambda)} .
$$

Definition 3.2. An element $\alpha \in \prod_{\lambda \in\left\{\begin{array}{c}\infty \\ l\end{array}\right\}} A$ is called a class if the polynomials $\alpha \mid \lambda$ for all $\lambda \in\left\{\begin{array}{c}\infty \\ l\end{array}\right\}$ have bounded total degree in the variables $y_{1}, y_{2}, \ldots$ and if it satisfies the GKM (Goresky-Kottwitz-MacPherson) condition, that is:

If $\lambda, \lambda^{\prime}$ in $\left\{\begin{array}{c}\infty \\ l\end{array}\right\}$ differ in the positions $i$ and $j$ only, the element $\alpha|\lambda-\alpha| \lambda^{\prime}$ is divisible by $y_{i}-y_{j}$.

It is clear that the classes in $\prod_{\lambda \in\left\{\begin{array}{c}\infty \\ l\end{array}\right\}} A$ form an $A$-algebra with coordinatewise addition and multiplication. We denote this algebra by $H(l)$.

A class $\alpha \in \prod_{\lambda \in\left\{\begin{array}{c}\infty \\ l\end{array}\right\}} A$ is a Schubert class corresponding to $\lambda$ in $\left\{\begin{array}{c}\infty \\ l\end{array}\right\}$ if it satisfies the following three conditions:

(1) $\alpha \mid \lambda=\prod_{(i, j) \in \operatorname{inv}(\lambda)}\left(y_{j}-y_{i}\right)$.

(2) If $\alpha \mid \lambda^{\prime} \neq 0$ then $\lambda^{\prime} \geq \lambda$.

(3) For all $\mu \in\left\{\begin{array}{c}\infty \\ l\end{array}\right\}$ the element $\alpha \mid \mu$ is homogeneous of degree $l(\lambda)$ in $y_{1}, y_{2}, \ldots$

The ring $H(l)$ can be thought of as the formal generalized analog of the localized equivariant cohomology of the infinite grassmannian described in the introduction, with one torus fixed point for each partition. In the following result we give the exact connection between classes and symmetric polynomials. Observe that the proof of assertion (2) is modelled after the proof of Proposition 1 in [Knutson and Tao 2003].

Proposition 3.3. Let $A=\mathbb{Z}\left[y_{1}, y_{2}, \ldots\right]$ be the polynomial ring in the independent variables $y_{1}, y_{2}, \ldots$ over $\mathbb{Z}$. Then: 
(1) The image of the homomorphism $\sigma^{\text {equ }}: A\left[T_{1}, \ldots, T_{l}\right]^{\mathrm{sym}} \rightarrow \prod_{\lambda \in\left\{\begin{array}{c}\infty \\ l\end{array}\right\}}$ A consists of classes, that is, $\sigma^{\mathrm{equ}}$ induces an A-algebra homomorphism

$$
\sigma: A\left[T_{1}, \ldots, T_{l}\right]^{\mathrm{sym}} \rightarrow H(l) .
$$

Moreover, the images $S_{b}=\sigma\left(s_{b}\left(T_{1}, \ldots, T_{l} \mid y\right)\right)$ of the factorial Schur functions are Schubert classes corresponding to $\lambda(b)$, for each partition $b: b_{1} \geq$ $\cdots \geq b_{l} \geq 0$ in $\left\{\begin{array}{c}\infty \\ l\end{array}\right\}_{\mathscr{P}}$.

(2) Let $\alpha$ be a class. Then $\alpha$ is a linear combination, with coefficients in $A$, of the Schubert classes $S_{b}=\sigma\left(s_{b}\left(T_{1}, \ldots, T_{l} \mid y\right)\right)$ for partitions $b: b_{1} \geq \cdots \geq b_{l} \geq 0$ in $\left\{\begin{array}{c}\infty \\ l\end{array}\right\}_{\mathscr{P}}$. In particular $\sigma$ is surjective.

If all the elements $\alpha \mid \lambda$ are in $\mathbb{Z}\left[y_{1}, \ldots, y_{n}\right]$ for some $n$, the coefficients are in $\mathbb{Z}\left[y_{1}, \ldots, y_{n}\right]$, and if $\sigma^{\mathrm{equ}}\left(s_{b}\left(T_{1}, \ldots, T_{l} \mid y\right)\right)$ has a non-zero coefficient then $b \in\left\{\begin{array}{c}\infty \\ l\end{array}\right\}_{k}$ with $k=n-l$.

Proof. It is clear that for $g \in A\left[T_{1}, \ldots, T_{l}\right]^{\text {sym }}$ the elements $\sigma^{\text {equ }}(g) \mid \lambda$ are of total degree at most equal to the total degree of $g$ plus the total degree of the coefficients. Moreover, it follows from Theorem 2.5(1) that $\sigma^{\text {equ }}(g)$ satisfies the GKM condition. Hence $\sigma^{\text {equ }}(g)$ is a class. That $S_{b}$ is a Schubert class follows from Theorem 2.4. Thus we have proved assertion (1).

We next prove assertion (2). Let $\alpha$ be a non-zero class. Let $\mu$ be minimal in the support of $\alpha$, that is, $\mu$ is minimal such that $\alpha \mid \mu \neq 0$. It follows from the GKM condition that $\alpha \mid \mu$ is a multiple $\beta$ of $\prod_{(i, j) \in \operatorname{inv}(\mu)}\left(y_{j}-y_{i}\right)$. Moreover, if $\alpha \mid \mu$ is in $\mathbb{Z}\left[y_{1}, \ldots, y_{n}\right]$ we must have $b(\mu)_{1}+l \leq n$, that is, $\mu \in\left\{\begin{array}{c}\infty \\ l\end{array}\right\}_{k}$ and $\beta \in \mathbb{Z}\left[y_{1}, \ldots, y_{n}\right]$. Theorem 2.4(1) implies that $\alpha\left|\mu=\beta \sigma^{\text {equ }}\left(s_{b(\mu)}\left(T_{1} \ldots, T_{l} \mid y\right)\right)\right| \mu$ and Theorem 2.4(2) that $\mu$ is not in the support of $\alpha-\beta \sigma^{\text {equ }}\left(s_{b(\mu)}\left(T_{1}, \ldots, T_{l} \mid y\right)\right)$ and that no element smaller than $\mu$ is in the support. Continuing this process we can successively reduce the support upwards. By the definition of a class the total degrees of $\alpha \mid \lambda$ for all $\lambda \in\left\{\begin{array}{c}\infty \\ l\end{array}\right\}$ are bounded. Thus the process must end. Moreover, in the process we have that if $\alpha \mid \lambda \in \mathbb{Z}\left[y_{1}, \ldots, y_{n}\right]$ for all $\lambda \in\left\{\begin{array}{c}\infty \\ l\end{array}\right\}$ then the coefficients $\beta$ are in $\mathbb{Z}\left[y_{1}, \ldots, y_{n}\right]$ and the $\mu$ involved are in $\left\{\begin{array}{c}\infty \\ l\end{array}\right\}_{k}$. Hence assertion (2) holds.

The correspondence between the equivariant Schubert calculus of Section 1 and the generalization of the Goresky-Kottwitz-MacPherson description mentioned above (see also [Knutson and Tao 2003], [Arabia 1989] and [Kostant and Kumar 1986]) is given by the following results.

Theorem 3.4. (1) The homomorphism $\sigma: A\left[T_{1}, \ldots, T_{l}\right]^{\mathrm{sym}} \rightarrow H(l)$ of Proposition 3.3 is an A-algebra isomorphism.

(2) The Schubert classes $S_{\lambda}$ for $\lambda \in\left\{\begin{array}{c}\infty \\ l\end{array}\right\}$ form a basis for the A-module of classes.

(3) $S_{\lambda}$ is the unique Schubert class belonging to $\lambda$. 
Proof. Let $g \in A\left[T_{1}, \ldots, T_{l}\right]^{\mathrm{sym}}$. The elements $(T \mid y)^{h_{1}+l-1} \wedge \cdots \wedge(T \mid y)^{h_{l}}$ for all partitions $h_{1} \geq \cdots \geq h_{l} \geq 0$ form a basis for $\bigwedge_{A}^{l} A[T]$. In particular, it follows from Proposition 1.5 that $g$ is the image by $\sigma^{\text {sym }}$ of a unique element $\sum_{h \in \mathscr{I}} z_{h}\left((T \mid y)^{h_{1}+l-1} \wedge \cdots \wedge(T \mid y)^{h_{l}}\right)$ with $z_{h} \in A$, and where the sum is over a finite set of partitions in $\left\{\begin{array}{c}\infty \\ l\end{array}\right\}$. Hence it follows from Theorem 1.2(1) and (3) that $g=\sum_{h \in \mathcal{I}} z_{h} s_{h}\left(T_{1}, \ldots, T_{l} \mid y\right)$ and that the elements $s_{b}\left(T_{1}, \ldots, T_{l} \mid y\right)$ for $b \in\left\{\begin{array}{c}\infty \\ l\end{array}\right\}_{\mathscr{P}}$ form an $A$-basis for $A\left[T_{1}, \ldots, T_{l}\right]^{\mathrm{sym}}$. If $\sigma^{\text {equ }}(g)=0$ it follows from Theorem 2.5(2) that $z_{h}=0$ for all $h \in \Phi$. Thus $\sigma^{\text {equ }}$ is injective. The $A$-algebra homomorphism $\sigma^{\text {equ }}$ maps $A\left[T_{1}, \ldots, T_{l}\right]^{\mathrm{sym}}$ onto classes by Proposition 3.3(2). Thus we have proved assertion (1).

Since we just proved that the classes $s_{b}\left(T_{1}, \ldots, T_{l} \mid y\right)$ for $b$ in $\left\{\begin{array}{c}\infty \\ l\end{array}\right\}_{\mathscr{P}}$ form a basis for $A\left[T_{1}, \ldots, T_{l}\right]^{\mathrm{sym}}$ assertion (2) follows from (1) and the definition of $S_{\lambda}$.

Assertion (3) follows easily from Theorem 2.5(3) and the definition of $S_{\lambda}$.

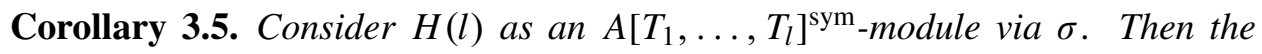
composite homomorphism

$$
\sigma \sigma^{\mathrm{sym}}: \bigwedge_{A}^{l} A[T] \rightarrow H(l)
$$

determined by mapping

$$
(T \mid y)^{b_{1}+l-1} \wedge \cdots \wedge(T \mid y)^{b_{l}}=s_{b}\left(T_{1}, \ldots, T_{l} \mid y\right)\left((T \mid y)^{l-1} \wedge \cdots \wedge(T \mid y)^{0}\right) \quad \text { to } \quad S_{\lambda(b)},
$$

for each partition $b: b_{1} \geq \cdots \geq b_{l} \geq 0$, is an isomorphism of $A\left[T_{1}, \ldots, T_{l}\right]^{\mathrm{sym}}$ modules.

Proof. The proposition immediately follows from Proposition 1.5 and from assertion (1) of the theorem.

\section{Pieri's formula}

Let $A$ be an arbitrary ring. From the action of $A\left[T_{1}, \ldots, T_{l}\right]^{\text {sym }}$ on $\bigwedge_{A}^{l} A[T]$ we obtain that the product $s_{h} T^{h_{1}} \wedge \cdots \wedge T^{h_{l}}$, where $s_{h}$ is the $h$-th complete symmetric function in $T_{1}, \ldots, T_{l}$ and $h_{1} \geq \cdots \geq h_{l} \geq 0$ is a partition, can be expressed as a linear combination of elements $T^{j_{1}} \wedge \cdots \wedge T^{j_{l}}$ with coefficients in $A\left[T_{1}, \ldots, T_{l}\right]^{\mathrm{sym}}$, where $j_{1} \geq \cdots \geq j_{l} \geq 0$ is a partition such that $j_{1}+\cdots+j_{l}=$ $h_{1}+\cdots+h_{l}+h$. After suitable cancellations the resulting formula is called Pieri's formula. In Sections 1 and 2 we have seen how the multiplication can be expressed in terms of factorial Schur functions. Here we shall give the explicit calculations of $s_{h 0 \ldots 0}\left(T_{1}, \ldots, T_{l} \mid y\right)\left((T \mid y)^{h_{1}} \wedge \cdots \wedge(T \mid y)^{h_{l}}\right)$ in $\bigwedge_{A}^{l} A[T]$ and perform the necessary simplifications to obtain the equivariant Pieri formula. 
Using the isomorphism in Corollary 3.5 the calculations and the resulting formulas can be translated into the algebra $H(l)$. There we obtain an explicit expression for the coordinatewise product $s_{h 0 \ldots 0} s_{h}\left(T_{1}, \ldots, T_{l} \mid y\right)$ as linear combinations of elements $s_{i_{1}, \ldots, i_{l}}\left(T_{1}, \ldots, T_{l} \mid y\right)$ with coefficients in $A\left[T_{1}, \ldots, T_{l}\right]^{\mathrm{sym}}$ where $i_{1}+\cdots+i_{l}=b_{1}+\cdots+b_{l}+h$, the coefficients are those of [Knutson and Tao 2003]. After the appropriate cancellations of terms we obtain the Pieri formula in $H(l)$. The results are direct translations of those in $\bigwedge_{A}^{l} A[T]$ and we therefore do not repeat them.

Similar calculations to those performed in this section were first made by Santiago in [Santiago 2006] (see also [Gatto and Santiago 2009; Laksov and Thorup 2009]). The formula specializes to those of [Mihalcea 2006; 2008]. A different approach to Pieri's formula can be found in [Robinson 2002].

To make the expressions of the calculations more transparent we simplify the notation somewhat.

For independent variables $T_{1}, \ldots, T_{l}$ over $A$ we denote by

$$
s_{h}=s_{h}\left(T_{1}, \ldots, T_{l}\right)
$$

the $h$-th complete symmetric function in $T_{1}, \ldots, T_{l}$, and by $s_{h}\left(y_{1}, \ldots, y_{l}\right)$ its value at $y_{1}, y_{2}, \ldots, y_{l} \in A$. Similarly, for elements $y_{1}, y_{2}, \ldots$ in $A$ and for any $h$ and $m$ we denote by

$$
c_{h}\left(y_{1}, \ldots, y_{m}\right)
$$

the value at $y_{1}, \ldots, y_{m}$ of the $h$-th elementary symmetric function in $m$ variables. We write

$$
g_{h}=(T \mid y)^{h}=\left(T-y_{1}\right) \cdots\left(T-y_{h}\right)
$$

and let

$$
\begin{aligned}
S_{h 0 \cdots 0}(T \mid y) & =s_{h 0 \cdots 0}\left(T_{1}, \ldots, T_{l} \mid y\right) \\
& =\operatorname{Res}\left((T \mid y)^{h+l-1} / Q(T),(T \mid y)^{l-2} / Q(T), \ldots,(T \mid y)^{0} / Q(T)\right)
\end{aligned}
$$

be the $h$-th factorial Schur function where $h \geq 0 \geq \cdots \geq 0$ is in $\left\{\begin{array}{c}\infty \\ l\end{array}\right\}_{\mathscr{P}}$.

Lemma 4.1. We have

$$
g_{h+l-1} \wedge g_{l-2} \wedge \cdots \wedge g_{0}=\sum_{j=0}^{h}(-1)^{j} s_{h-j} c_{j}\left(y_{1}, \ldots, y_{h+l-1}\right)\left(g_{l-1} \wedge \cdots \wedge g_{0}\right),
$$

where $c_{j}\left(y_{1}, \ldots, y_{h+l-1}\right)$ is the $j$-th elementary symmetric function in the variables $y_{1}, \ldots, y_{h+l-1}$. Moreover,

$$
s_{h 0 \cdots 0}(T \mid y)=\sum_{j=0}^{h}(-1)^{j} s_{h-j} c_{j}\left(y_{1}, \ldots, y_{h+l-1}\right) .
$$


Proof. We develop $g_{h+l-1}, g_{l-2}, \ldots, g_{0}$ in powers of the variable $T$ and obtain $g_{h+l-1} \wedge g_{l-2} \wedge \cdots \wedge g_{0}$

$$
=\left(\sum_{j=0}^{h+l-1}(-1)^{j} c_{j}\left(y_{1}, \ldots, y_{h+l-1}\right) T^{h+l-1-j}\right) \wedge T^{l-2} \wedge \cdots \wedge T^{0} .
$$

Theorem 1.2(3) implies that $T^{h+l-1-j} \wedge T^{l-2} \wedge \cdots \wedge T^{0}=s_{h-j}\left(T^{l-1} \wedge \cdots \wedge T^{0}\right)$. Thus

$$
g_{h+l-1} \wedge g_{l-2} \wedge \cdots \wedge g_{0}=\sum_{j=0}^{h+l-1}(-1)^{j} s_{h-j} c_{j}\left(y_{1}, \ldots, y_{h+l-1}\right)\left(T^{l-1} \wedge \cdots \wedge T^{0}\right),
$$

which gives the first part of the lemma since $s_{h-j}=0$ for $j>k$, as required.

To obtain the last part of the lemma it suffices to compare the first equation of the lemma with the equation $g_{h+l-1} \wedge g_{l-2} \wedge \cdots \wedge g_{0}=s_{h 0 \cdots 0}(T \mid y)\left(g_{l-1} \wedge \cdots \wedge g_{0}\right)$ of Theorem 1.2(3).

Lemma 4.2. We have

$$
T^{h} g_{i}=\sum_{j=0}^{h} s_{h-j}\left(y_{i+1}, \ldots, y_{i+j+1}\right) g_{i+j} .
$$

Proof. We prove the equation by induction on $h$. It holds trivially for $h=0$. Assume it holds for $h>0$. From $T g_{i}=g_{i+1}+y_{i+1} g_{i}$ and the induction hypothesis we get

$$
\begin{aligned}
T^{h+1} g_{i}= & \sum_{j=0}^{h} s_{h-j}\left(y_{i+1}, \ldots, y_{i+j+1}\right)\left(g_{i+j+1}+y_{i+j+1} g_{i+j}\right) \\
= & \sum_{j=1}^{h+1} s_{h+1-j}\left(y_{i+1}, \ldots, y_{i+j}\right) g_{i+j}+\sum_{j=0}^{h} s_{h-j}\left(y_{i+1}, \ldots, y_{i+j+1}\right) y_{i+j+1} g_{i+j} \\
= & s_{0}\left(y_{i+1}, \ldots, y_{i+h+1}\right) g_{i+h+1} \\
& \quad+\sum_{j=1}^{h}\left(s_{h+1-j}\left(y_{i+1}, \ldots, y_{i+j}\right)+s_{h-j}\left(y_{i+1}, \ldots, y_{i+j+1}\right) y_{i+j}\right) g_{i+j} \\
& \quad+s_{h}\left(y_{i+1}\right) y_{i+1} g_{i} \\
= & g_{i+h+1}+\sum_{j=1}^{h} s_{h+1-j}\left(y_{i+1}, \ldots, y_{i+j+1}\right) g_{i+j}+s_{h+1}\left(y_{i+1}\right) g_{i} \\
= & \sum_{j=0}^{h+1} s_{h+1-j}\left(y_{i+1}, \ldots, y_{i+j+1}\right) g_{i+j} .
\end{aligned}
$$

Lemma 4.3. Let $j_{1}, \ldots, j_{l}, h_{1}, \ldots, h_{l}, h$ be non-negative integers with $j_{1}+\cdots+$ $j_{l} \leq h$. Then

$$
\begin{aligned}
\sum_{i_{1}+\cdots+i_{l}=h} s_{i_{1}-j_{1}}\left(y_{h_{1}+1}, \ldots, y_{h_{1}+j_{1}+1}\right) \cdots s_{i_{l}-j_{l}}\left(y_{h_{l}+1}, \ldots, y_{h_{l}+j_{l}+1}\right) \\
=s_{h-j_{1}-\cdots-j_{l}}\left(y_{h_{1}+1}, \ldots, y_{h_{1}+j_{1}+1}, \ldots, y_{h_{l}+1}, \ldots, y_{h_{l}+j_{l}+1}\right) .
\end{aligned}
$$


Proof. It is clear that all the monomials on the left-hand side of the equation of the lemma appear in the right-hand side. Conversely, consider a monomial that appears on the right-hand side with a contribution of degree $k_{i}$ from the variables $y_{h_{i}+1}, \ldots, y_{h_{i}+j_{i}+1}$ for $i=1, \ldots, l$. Then $k_{1}+\cdots+k_{l}=h-j_{1}-\cdots-j_{l}$. Let $i_{1}:=h-j_{2}-\cdots-j_{l}-k_{2}-\cdots-k_{l}$. Then $i_{1}-j_{1}=k_{1}$. Correspondingly we define $i_{2}, \ldots, i_{l}$ such that $i_{p}-j_{p}=k_{p}$ for $p=1, \ldots, l$. The monomial that we consider will then be the product of monomials in $y_{h_{p}+1}, \ldots, y_{h_{p}+j_{p}+1}$ of degree $i_{p}-j_{p}=k_{p}$ for $p=1, \ldots, l$, and thus appear on the left-hand side of the equation in the lemma.

Proposition 4.4. Let $h_{1}, \ldots, h_{l}, h$ be non-negative integers. Then

$$
\begin{aligned}
& s_{h}\left(g_{h_{1}} \wedge \cdots \wedge g_{h_{l}}\right)=\sum_{i=0}^{h} \sum_{j_{1}+\cdots+j_{l}=h-i} \\
& s_{i}\left(y_{h_{1}+1}, \ldots, y_{h_{1}+j_{1}+1}, \ldots, y_{h_{l}+1}, \ldots, y_{h_{l}+j_{l}+1}\right)\left(g_{h_{1}+j_{1}} \wedge \cdots \wedge g_{h_{l}+j_{l}}\right) .
\end{aligned}
$$

Proof. By definition $\omega:=s_{h} g_{h_{1}} \wedge \cdots \wedge g_{h_{l}}=\sum_{i_{1}+\cdots+i_{l}=h} T^{i_{1}} g_{h_{1}} \wedge \cdots \wedge T^{i_{l}} g_{h_{l}}$. It follows from Lemma 4.2 that

$$
\begin{aligned}
& \omega=\sum_{i_{1}+\cdots+i_{l}=h} \sum_{j_{1}=0}^{i_{1}} \cdots \sum_{j_{l}=0}^{i_{l}} s_{i_{1}-j_{1}}\left(y_{h_{1}+1}, \cdots, y_{h_{1}+j_{1}+1}\right) \cdots \\
& s_{i_{l}-j_{l}}\left(y_{h_{l}+1}, \ldots y_{h_{l}+j_{l}+1}\right)\left(g_{h_{1}+j_{1}} \wedge \cdots \wedge g_{h_{l}+j_{l}}\right) .
\end{aligned}
$$

Since $s_{i_{p}-j_{p}}\left(y_{h_{p}+1}, \ldots, y_{h_{p}+j_{p}+1}\right)=0$ when $j_{p}>i_{p}$ we obtain

$$
\begin{aligned}
& \omega=\sum_{i_{1}+\cdots+i_{l}=h} \sum_{j_{1}+\cdots+j_{l}=0}^{h} s_{i_{1}-j_{1}}\left(y_{h_{1}+1}, \ldots, y_{h_{1}+j_{1}+1}\right) \cdots \\
& s_{i_{l}-j_{l}}\left(y_{h_{l}+1}, \ldots, y_{h_{l}+j_{l}+1}\right)\left(g_{h_{1}+j_{1}} \wedge \cdots \wedge g_{h_{l}+j_{l}}\right) .
\end{aligned}
$$

Exchanging the order of summation we obtain by Lemma 4.3

$$
\begin{aligned}
\omega=\sum_{j_{1}+\cdots+j_{l}=0}^{h} s_{h-j_{1}-\cdots-j_{l}}\left(y_{h_{1}+1}, \ldots, y_{h_{1}+j_{1}+1}, \ldots, y_{h_{l}+1}, \ldots, y_{h_{l}+j_{l}+1}\right) & \left(g_{h_{1}+j_{1}} \wedge \cdots \wedge g_{h_{l}+j_{l}}\right),
\end{aligned}
$$

that immediately gives the equation of the proposition.

Theorem 4.5 (Pieri's formula). Let $h_{1} \geq \cdots \geq h_{l} \geq 0$ be a partition and let $h$ be a non-negative integer. Then

$$
\begin{aligned}
s_{h}\left(g_{h_{1}} \wedge \cdots \wedge g_{h_{l}}\right) & =\sum_{i=0}^{h} \sum_{\left(j_{1}, \ldots, j_{l}\right) \in \mathscr{F}_{h-i}} \\
& s_{i}\left(y_{h_{1}+1}, \ldots, y_{h_{1}+j_{1}+1}, \ldots, y_{h_{l}+1}, \ldots, y_{h_{l}+j_{l}+1}\right)\left(g_{h_{1}+j_{1}} \wedge \cdots \wedge g_{h_{l}+j_{l}}\right),
\end{aligned}
$$


where $\mathscr{S}_{h-i}$ is the collection of all l-tuples $\left(j_{1}, \ldots, j_{l}\right)$ such that $j_{1}+\cdots+j_{l}=h-i$ and $j_{1}+h_{1} \geq h_{1}>j_{2}+h_{2} \geq h_{2}>\cdots>j_{l}+h_{l} \geq h_{l}$. We also have

$$
\begin{aligned}
s_{h 0 \ldots 0}(T \mid y)\left(g_{h_{1}} \wedge \cdots \wedge g_{h_{l}}\right) & \\
= & \sum_{j=0}^{h} \sum_{i=0}^{h} \sum_{\left(j_{1}, \ldots, j_{l}\right) \in \mathscr{F}_{h-i}}^{h}(-1)^{j} c_{j}\left(y_{1}, \ldots, y_{h+l-1}\right) \\
& s_{i}\left(y_{h_{1}+1}, \ldots, y_{h_{1}+j_{1}+1}, \ldots, y_{h_{l}+1}, \ldots, y_{h_{l}+j_{l}+1}\right)\left(g_{h_{1}+j_{1}} \wedge \cdots \wedge g_{h_{l}+j_{l}}\right),
\end{aligned}
$$

Proof. Let $s_{i}\left(g_{h_{1}+j_{1}} \wedge \cdots \wedge g_{h_{l}+j_{l}}\right)$ be a term on the right-hand side of the equation of Proposition 4.4. Assume that $h_{p}+j_{p} \geq h_{p-1}$ for some $p$. If $h_{p}+j_{p}=h_{p-1}+j_{p-1}$ the term is zero. Assume, on the other hand, that $j_{p-1}^{\prime}:=h_{p}+j_{p}-h_{p-1} \neq j_{p-1}$, and let $j_{p}^{\prime}:=h_{p-1}+j_{p-1}-h_{p}$. Then $j_{p-1}^{\prime} \geq 0$ and $j_{p}^{\prime}=h_{p-1}-h_{p}+j_{p-1} \geq 0$. Moreover we have $j_{p-1}^{\prime}+j_{p}^{\prime}=j_{p-1}+j_{p}$, and $j_{p}^{\prime} \neq j_{p}$ because $j_{p-1}^{\prime} \neq j_{p-1}$. On the right-hand side of the sum in Proposition 4.4 we thus have two terms

$$
\begin{aligned}
& s_{i}\left(\ldots, y_{h_{p-1}+1}, \ldots, y_{h_{p-1}+j_{p-1}+1}, y_{h_{p}+1}, \ldots, y_{h_{p}+j_{p}+1}, \ldots\right) \\
& \left(\cdots \wedge g_{h_{p-1}+j_{p-1}} \wedge g_{h_{p}+j_{p}} \wedge \ldots\right) \\
+s_{i} & \left(\ldots, y_{h_{p-1}+1}, \ldots, y_{h_{p-1}+j_{p-1}^{\prime}+1}, y_{h_{p}+1}, \ldots, y_{h_{p}+j_{p}^{\prime}+1}, \ldots\right) \\
& \left(\cdots \wedge g_{h_{p-1}+j_{p-1}^{\prime}} \wedge g_{h_{p}+j_{p}^{\prime}} \wedge \ldots\right) .
\end{aligned}
$$

Since $h_{p-1}+j_{p-1}=h_{p}+j_{p}^{\prime}$ and $h_{p}+j_{p}=h_{p-1}+j_{p-1}^{\prime}$ and the $s_{i}$ are symmetric in $y_{h_{l}+1}, \ldots, y_{h_{1}+j_{1}+l}$, these two terms cancel. In Proposition 4.4 there remain only the terms in Pieri's formula.

The last formula follows from the first and Lemma 4.1.

\section{Acknowledgments}

We thank W. Fulton and L. C. Mihalcea for valuable comments, and W. Fulton for pointing out the interest in finding the connections between our generalized Schubert calculus and the Schubert calculus in equivariant cohomology, and thereby initiating the present work.

\section{References}

[Arabia 1989] A. Arabia, "Cohomologie $T$-équivariante de la variété de drapeaux d'un groupe de Kac-Moody”, Bull. Soc. Math. France 117:2 (1989), 129-165. MR 90i:32042 Zbl 0706.57024

[Billey 1999] S. C. Billey, "Kostant polynomials and the cohomology ring for G/B", Duke Math. J. 96:1 (1999), 205-224. MR 2000a:14060 Zbl 0980.22018

[Fulton 1997] W. Fulton, Young tableaux, with applications to representation theory and geometry, London Math. Soc. Student Texts 35, Cambridge Univ. Press, 1997. MR 99f:05119 Zbl 0878.14034 [Fulton 1998] W. Fulton, Intersection theory, 2nd ed., Ergebnisse der Mathematik (3) 2, Springer, Berlin, 1998. MR 99d:14003 Zbl 0885.14002 
[Fulton 2007] W. Fulton, "Equivariant cohomology in algebraic geometry", notes of Eilenberg lectures (taken by David Anderson), Columbia University, New York, spring 2007, Available at http:// www.math.Isa.umich.edu/ dandersn/eilenberg/index.html.

[Gatto 2005] L. Gatto, "Schubert calculus via Hasse-Schmidt derivations", Asian J. Math. 9:3 (2005), 315-321. MR 2006m:14075 Zbl 1099.14045

[Gatto and Santiago 2006] L. Gatto and T. Santiago, "Equivariant Schubert calculus", preprint, 2006. arXiv math/0703445

[Gatto and Santiago 2009] L. Gatto and T. Santiago, "Schubert calculus on a Grassmann algebra", Canad. Math. Bull. 52:2 (2009), 200-212. MR 2512308 Zbl 1168.14039

[Kempf and Laksov 1974] G. Kempf and D. Laksov, "The determinantal formula of Schubert calculus", Acta Math. 132 (1974), 153-162. MR 49 \#2773 Zbl 0295.14023

[Knutson and Tao 2003] A. Knutson and T. Tao, "Puzzles and (equivariant) cohomology of Grassmannians", Duke Math. J. 119:2 (2003), 221-260. MR 2006a:14088 Zbl 1064.14063

[Kostant and Kumar 1986] B. Kostant and S. Kumar, "The nil Hecke ring and cohomology of $G / P$ for a Kac-Moody group G”, Adv. in Math. 62:3 (1986), 187-237. MR 88b:17025b Zbl 0641.17008 [Lakshmibai et al. 2006] V. Lakshmibai, K. N. Raghavan, and P. Sankaran, "Equivariant Giambelli and determinantal restriction formulas for the Grassmannian", Pure Appl. Math. Q. 2:3, part 1 (2006), 699-717. MR 2007h:14084 Zbl 1105.14065

[Laksov 2008] D. Laksov, "Schubert calculus and equivariant cohomology of Grassmannians", Adv. Math. 217:4 (2008), 1869-1888. MR 2009m:14082 Zbl 1136.14042

[Laksov and Thorup 2009] D. Laksov and A. Thorup, "Schubert calculus on Grassmannians and exterior powers”, Indiana Univ. Math. J. 58:1 (2009), 283-300. MR 2504412 Zbl 05553171

[Lascoux 2003] A. Lascoux, Symmetric functions and combinatorial operators on polynomials, CBMS Regional Conference Series in Mathematics 99, American Mathematical Society, Providence, 2003. MR 2005b:05217 Zbl 1039.05066

[Macdonald 1995] I. G. Macdonald, Symmetric functions and Hall polynomials, 2nd ed., Oxford University Press, New York, 1995. MR 96h:05207 Zbl 0824.05059

[Mihalcea 2006] L. Mihalcea, "Equivariant quantum Schubert calculus", Adv. Math. 203:1 (2006), 1-33. MR 2007c:14061 Zbl 1100.14045

[Mihalcea 2008] L. C. Mihalcea, "Giambelli formulae for the equivariant quantum cohomology of the Grassmannian”, Trans. Amer. Math. Soc. 360:5 (2008), 2285-2301. MR 2009e:14099 Zbl 1136.14046

[Molev and Sagan 1999] A. I. Molev and B. E. Sagan, "A Littlewood-Richardson rule for factorial Schur functions”, Trans. Amer. Math. Soc. 351:11 (1999), 4429-4443. MR 2000a:05212 Zbl 0972.05053

[Okun'kov and Ol'shanskiǔ 1997] A. Okun'kov and G. Ol'shanskiŭ, "Shifted Schur functions", Algebra i Analiz 9:2 (1997), 73-146. In Russian; translated in St. Petersburg Math. J. 9 (1997). MR 99f:05118

[Robinson 2002] S. Robinson, "A Pieri-type formula for $H_{T}^{*}\left(\mathrm{SL}_{n}(\mathbb{C}) / B\right)$ ", J. Algebra 249:1 (2002), 38-58. MR 2003b:14065 Zbl 1061.14060

[Santiago 2006] T. Santiago, Schubert calculus on a Grassmann algebra, Ph.D. thesis, Politechnico di Torino, 2006.

Communicated by Ravi Vakil

Received 2009-02-17 Revised 2009-06-26 Accepted 2009-08-06

laksov@math.kth.se KTH, Department of Mathematics, 10044 Stockholm, Sweden 